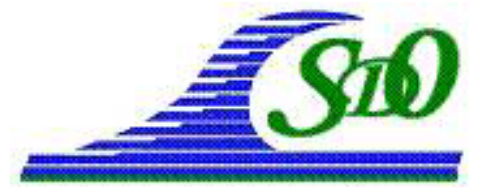

XI ìmes Journées Nationales Génie Côtier - Génie Civil

Les Sables d'Olonne, 22-25 juin 2010

DOI:10.5150/jngcgc.2010.064-R C Editions Paralia CFL

disponible en ligne - http://www.paralia.fr - available online

\title{
Typologie des barres d'avant-côte du golfe du Lion et impacts des ouvrages côtiers : apport de la technologie LIDAR
}

\author{
Nicolas ROBIN ${ }^{1}$, Raphaël CERTAIN ${ }^{1}$, Cyril VANROYE ${ }^{2}$, \\ Jean-Paul BARUSSEAU ${ }^{1}$, Frédéric BOUCHETTE ${ }^{3}$
}

1. Université de Perpignan Via Domitia, E.A. 3678 IMAGES, 52 av P. Alduy, 66860 Perpignan Cedex, France.

nicolas.robin@univ-perp.fr

2. Direction Régionale de l'Equipement du Languedoc-Roussillon, 520 Allée Henri II de Montmorency, 34064 Montpellier Cedex 2, France.

3. cc 51, Institut de Mathématique et de Modélisation, CNRS/Université Montpellier II, France.

\section{Résumé :}

Le golfe du lion est caractérisé par la présence d'un vaste ensemble de barres sédimentaires présentes sur l'avant-côte jusqu'à des profondeurs de $-8 \mathrm{~m} \mathrm{CM}$. Un premier inventaire régional de ces morphologies basé sur l'analyse planimétrique de photos aériennes a montré la diversité des configurations de barres (BARUSSEAU \& SAINT-GUILY, 1981). De nos jours, une analyse plus fine de la répartition des morphologies est possible grâce à l'utilisation d'une nouvelle technologie aéroportée, le LIDAR. L'objectif de ce travail est d'abord de proposer une actualisation de l'inventaire régional puis de mesurer l'influence des nombreux ouvrages côtiers sur les morphologies de barres.

\section{Mots clés :}

Barres sédimentaires - Golfe du Lion - LIDAR - Impact des ouvrages

\begin{abstract}
:
The gulf of Lion is characterized by the presence of a wide range of sedimentary offshore bars until $-8 \mathrm{~m}$ of depth. A first regional inventory of these morphological features based on planimetric analysis of aerial photos showed the diversity of bar pattern (BARUSSEAU \& SAINT-GUILY, 1981). Nowadays, a more detailed analysis of the distribution of morphologies is possible through the use of new airborne LIDAR technology. The objective of this work is first to propose an updating of the regional inventory and secondly to measure the influence of many coastal structures on the bar morphology.
\end{abstract}




\section{Introduction}

La présence de barres sédimentaires subtidales est très régulièrement observée sur les côtes sableuses. Elles peuvent prendre différentes formes (oblique, festonnée, rectiligne, une ou plusieurs barres) comme l'atteste les classifications disponibles dans la littérature (e.g. SHORT \& AAGAARD, 1993) basées essentiellement sur des paramètres morpho-hydrodynamiques à un instant $\mathrm{T}$. Ces observations sont généralement issues d'analyses de photographies aériennes parfois non simultanées ou de suivis bathymétriques de faible extension. Ces techniques traditionnelles peuvent alors "gommer" les hétérogénéités longitudinales des reliefs et masquer l'interface entre deux configurations de barres. Elles soulignent la difficulté de réaliser en peu de temps des levés de grande couverture. Ceci est dorénavant possible grâce à la technique aéroportée LIDAR (SHRESTHA et al., 2005).

L'objectif de ce travail est d'abord de proposer une actualisation de l'inventaire régional des barres accompli en 1981 (BARUSSEAU \& SAINT-GUILY, 1981), à partir d'un survol LIDAR réalisé fin août 2009, puis de mesurer l'influence des ouvrages côtiers (ouvrages portuaires de plaisance et ouvrages de défense) sur ces morphologies.

\section{Site d'étude}

Le golfe du Lion est une vaste unité déployant $200 \mathrm{~km}$ de linéaire côtier dans laquelle une grande variété de morphologies et d'environnements sont rencontrés. La relation entre la marée (0,3 $\mathrm{m}$ de marnage en vive-eau moyenne) et la houle $\left(0,8 \mathrm{~m}\right.$ de $\left.\mathrm{Hs}_{\text {moy }}\right)$ classe cette région dans un environnement dominé par les vagues (CERTAIN, 2002).

La dérive littorale est dirigée pour la partie sud du golfe du Lion (d'Argelès jusque dans le Narbonnais) du sud vers le nord et inversement pour la partie nord (du nord-est vers le sud-ouest). La présence de caps rocheux saillants (Mont Saint Clair à Sète, Cap d'Agde et Cap Leucate) provoque des interruptions totales ou partielles de la dérive littorale (figure 1). Transversalement, le profil présente une plage généralement étroite (quelques mètres à dizaines de mètres) et une avant-côte à barres avec plusieurs configurations (rectiligne ou festonné, une ou plusieurs barres), (BARUSSEAU \& SAINT-GUILY, 1981).

\section{Méthodologie}

Un survol LIDAR de l'ensemble de la côte sableuse du Languedoc-Roussillon a été effectué entre le 24 août et le 07 septembre 2009 à la demande de la Direction Régionale de l'Environnement, de l'Aménagement et du Logement LanguedocRoussillon (DREAL-LR) avec l'assistance technique et l'expertise du Service Hydrographique et Océanographique de la Marine (SHOM), (VANROYE, 2009). Cette période est choisie car elle correspond à un état stable du système après de longues semaines de temps calme. 
Le système employé par le groupement d'entreprise EUROSENSE et FUGRO LADS Corporation pour la réalisation du levé topo-bathymétrique est le LADS Mk II installé sur un avion Dash 8-202 volant entre 1200 et 2200 pieds. La fréquence du laser vert est de $900 \mathrm{~Hz}$ pour une résolution du levé minimale de $5 \mathrm{~m} * 5 \mathrm{~m}$ et une largeur de fauchée de $240 \mathrm{~m}$. Pour ce levé, l'espace entre les lignes de vol a été de $220 \mathrm{~m}$ permettant ainsi un recouvrement de $20 \mathrm{~m}$ entre lignes. Des lignes de vol transversales au trait de côte ont également été réalisées tous les $5000 \mathrm{~m}$. Les données récoltées s'étendent du cordon dunaire jusqu'à des profondeurs de $-20 \mathrm{~m}$. Au total, la superficie couverte est de $300 \mathrm{~km}^{2}$ avec 25 millions de points de mesure.

Trois zones de contrôle dont la bathymétrie est connue ont fait l'objet d'un survol systématique à chaque début et fin de mission journalière. La pré-validation des mesures a été réalisée pendant la mission avec une équipe de FUGRO LADS Corp., complétée par un traitement postérieur à Adélaïde et contrôlée par le SHOM. Deux stations GPS locales ont été installées dans le cadre de la campagne. La marée a fait l'objet d'un suivi spécifique au moyen de l'installation de deux observatoires temporaires (Port-la-Nouvelle et Port-Camargue) et de la fourniture des données du SHOM (PortVendres, Sète et Saintes-Maries de la Mer).

\section{Résultats}

\subsection{Configurations classiques}

L'étude à l'échelle régionale de la disposition des morphologies subtidales permet de délimiter deux unités du large vers la côte : une barre externe et un système interne constitué d'une barre interne et d'une barre de plage sous-marine inconstante et complexe.

Concernant la barre externe, l'étude de sa forme permet de décrire un compartimentage du golfe du Lion. Elle est festonnée au sud du cap Leucate (figures 1A et 1D) et linéaire au nord (figures $1 \mathrm{~A}$ et 1B). Les 81 festons identifiés dans le compartiment sud présentent une grande variabilité de leur longueur d'onde $(\Lambda)$ (d'Argelès à Canet : $\Lambda=357 \pm 125 \mathrm{~m}$; de Canet à Leucate : $\Lambda=475 \pm 167 \mathrm{~m}$ ). La longueur d'onde des festons semble être en relation avec l'éloignement de la barre à la côte (D) suivant la fonction suivante : $\mathrm{D}=0,55 \Lambda+53$ (coefficient de corrélation=0,84).

Concernant la barre interne, elle est généralement festonnée dans les deux compartiments (figure 1C) avec dans certains cas, une longueur d'onde tellement importante qu'elle peut être considérée comme quasi-rectiligne. La barre de plage sousmarine est présente occasionnellement et fournit des motifs très variés qui peuvent avoir une distribution périodique (figure 1D).

Le caractère nouveau apporté par l'imagerie LIDAR est de rendre possible le suivi continu de la position de la barre externe le long du littoral. On remarque à cet égard que son éloignement n'est pas constant. 
Thème 3 - Instrumentation, mesures, imagerie et télédétection

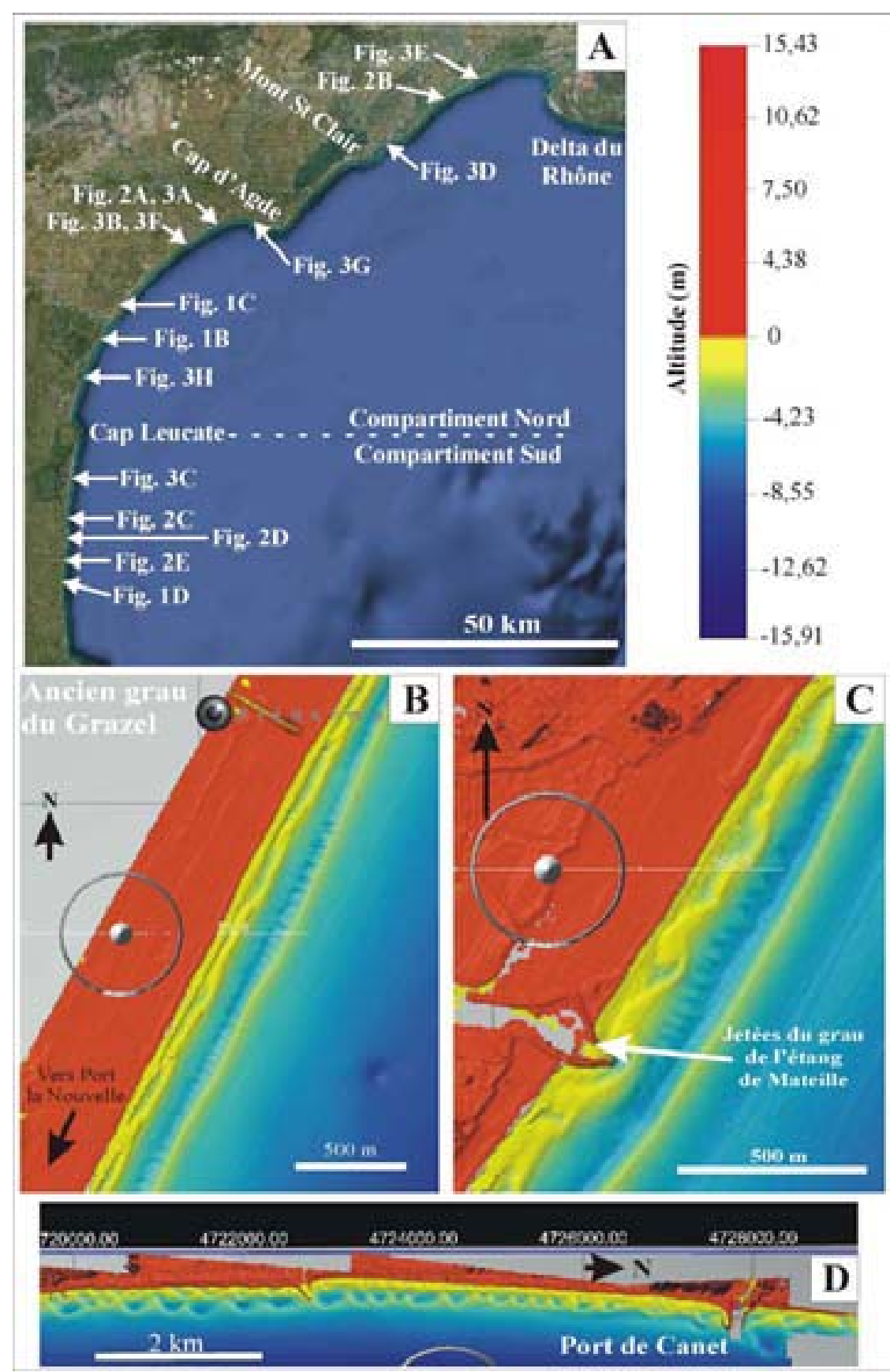

Figure 1. Localisation des différents zooms (a) et exemples de configurations classiques.

\subsection{Configurations complexes}

L'ensemble du système interne montre une grande variabilité d'aspects (figure 2) que l'on peut classer en fonction du plus ou moins grand degré d'organisation. Dans sa forme la mieux structurée, le système interne est complet et continu avec une barre interne (BI) festonnée et des éléments de la barre de plage sous-marine (BPSM) 


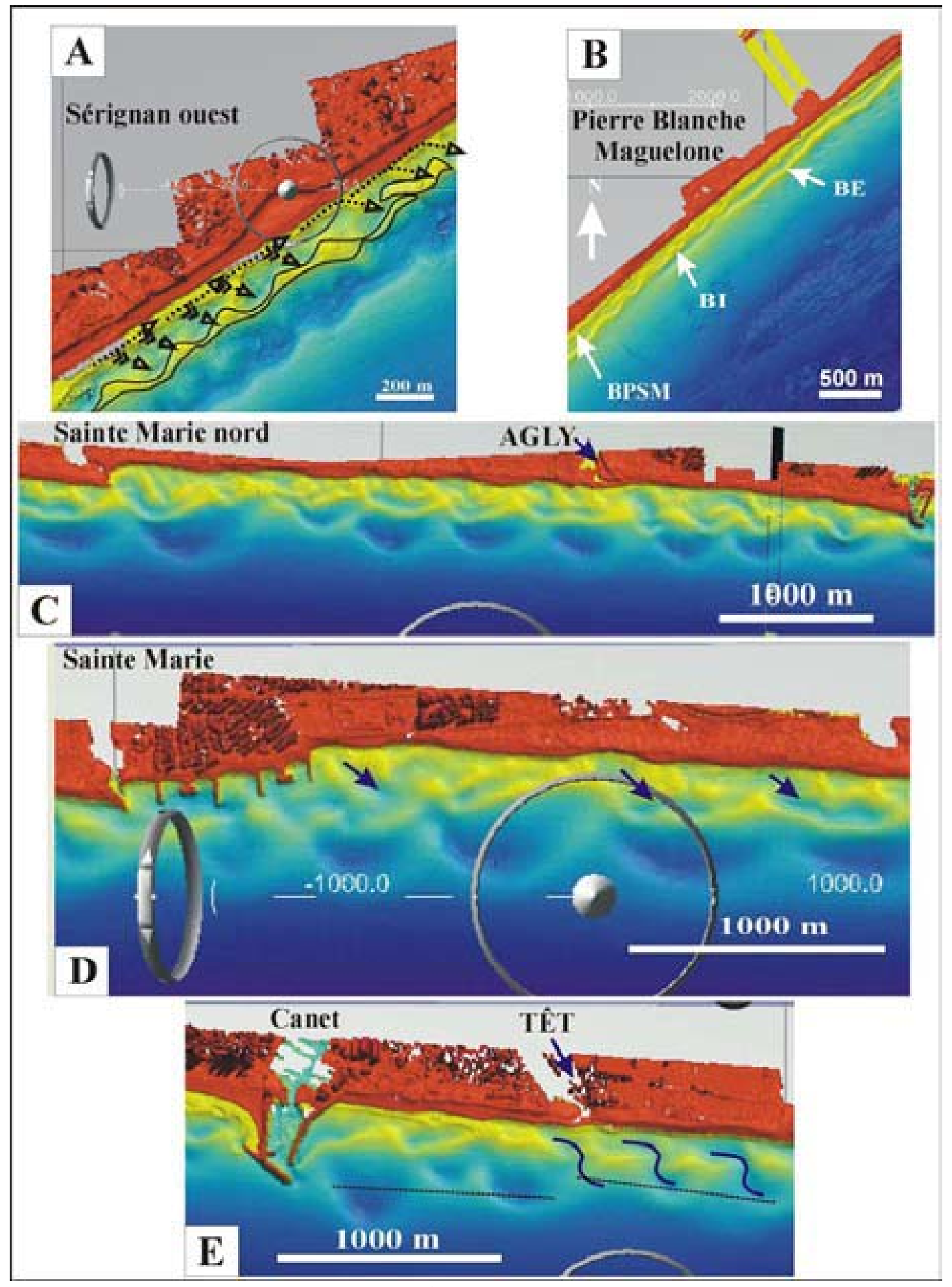

Figure 2. Exemples de configurations complexes. 
obliques (figure 2A). Dans certaines configurations, les deux éléments s'amalgament progressivement en un seul (figure $2 \mathrm{~B}$ ). La désorganisation progressive du système se manifeste d'abord par une irrégularité de la BPSM qui conserve cependant une certaine périodicité des dépocentres (figure 2C), puis par sa segmentation par des chenaux obliques drainant les courants d'arrachement vers le ventre de la barre interne du côté amont transit (figure 2D). Le stade final est sa disparition quasi-totale tandis que la barre interne est elle-même segmentée (figure 2E).

\subsection{Perturbations liées aux ouvrages et aux ports}

Différents types d'informations expriment la réaction du milieu à la perturbation du transit littoral par les ouvrages de défense contre la mer. Certaines d'entre elles sont d'ailleurs semblables aux effets induits par les obstacles naturels (embouchures, graus, promontoires rocheux). Les plus manifestes concernent la contraction du système des barres et son rapprochement du rivage (figures 3B, 3C). Les observations qui se rattachent à ce type d'influence sont abondantes dans le compartiment sud. Par exemple, à Saint-Cyprien, la barre externe se rapproche du rivage $(205 \mathrm{~m}$ au nord contre $280 \mathrm{~m}$ au sud). En aval-transit des jetées du grau de l'étang de Canet, la barre externe s'infléchit vers le rivage en l'espace de $3 \mathrm{~km}$ (140 m contre $215 \mathrm{~m})$. Dans le compartiment nord et en particulier à l'est de l'embouchure de l'Orb, il est assez difficile de séparer clairement les effets des ouvrages de ceux résultant de la pénurie sédimentaire qui en a provoqué l'édification. Ainsi, il ne semble pas possible de leur imputer les indications de carence sédimentaire qu'on y observe : apparition des fosses de lévigation asédimentaires (figure 3A); extension des affleurements du substratum (figure $3 \mathrm{E}$ ); et le caractère embryonnaire du système interne (figure 3D). L'imagerie LIDAR confirme cependant les effets profondément perturbateurs des grands aménagements (jetées portuaires) et permet d'évaluer l'influence des petits ouvrages (épis, brise-lames) sur le système. Elles concernent par exemple, l'affouillement autour des jetées (figures 3G et 3H), l'appréciation de l'état général des ouvrages et la mise en évidence de leur niveau de déstabilisation (blocs détachés en pied d'ouvrage - figure $3 \mathrm{~F} 1$ ) ou l'incidence de leur action sur la rétention sédimentaire (ruptures de pente de talus - figure 3F2).

\section{Discussion-Conclusion}

Dans le golfe du Lion, les différentes configurations décrites dans la classification de SHORT et AAGAARD (1993) se rencontrent simultanément dans les diverses morphologies observées, sans qu'on puisse invoquer une filiation temporelle. Leur proximité spatiale conduit à rechercher d'autres pistes pour en expliquer la variabilité. Le volume du disponible sédimentaire semble jouer un rôle non seulement sur l'ampleur des barres mais aussi sur leur organisation spatiale (nombre, forme et relations mutuelles). 


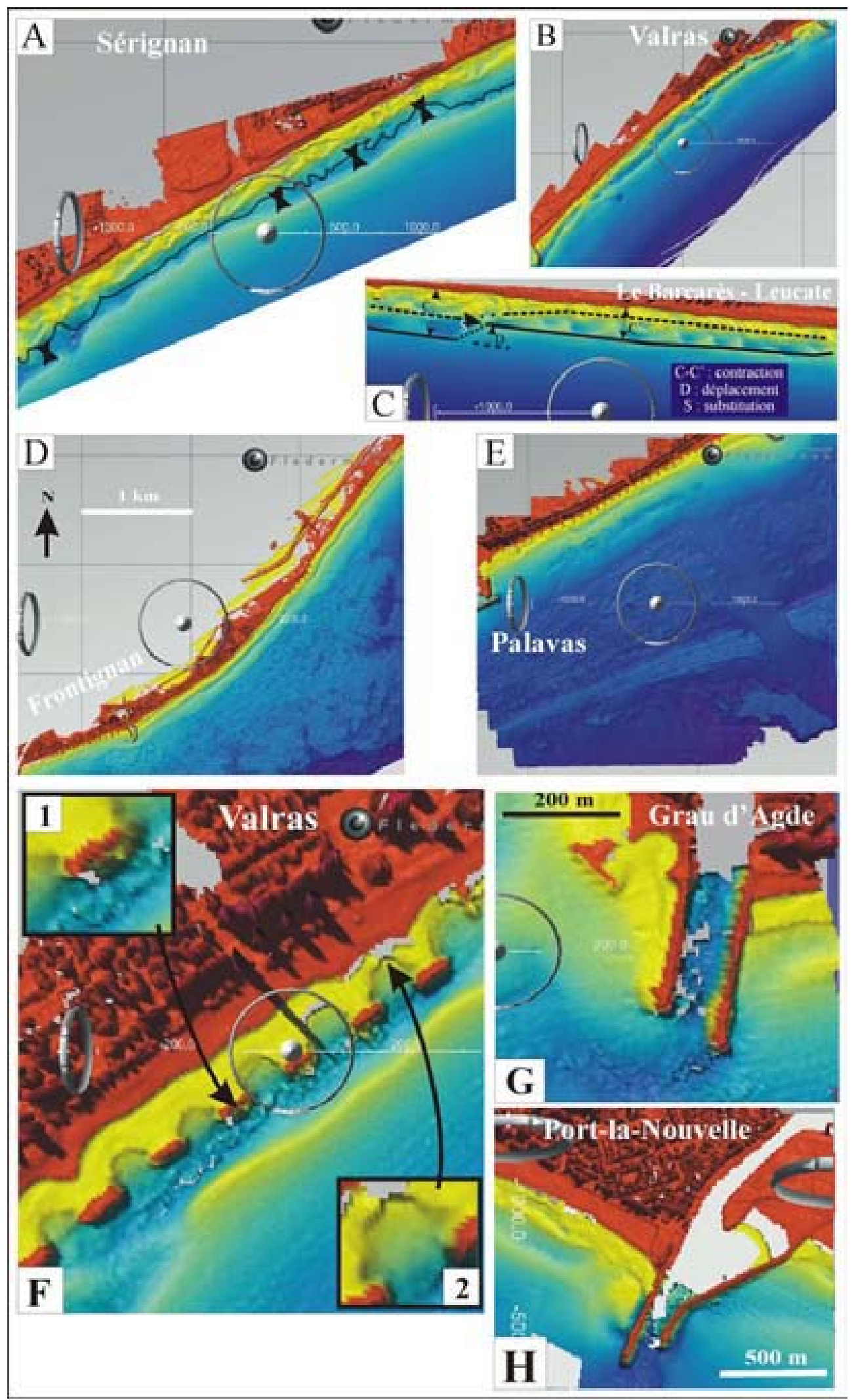

Figure 3. Exemples de perturbation des ouvrages côtiers. 
La typologie des barres pourrait donc se rattacher autant à leur histoire et aux conditions de l'apport sableux qu'à l'environnement énergétique de dépôt. Ces deux paramètres principaux restent encore à corréler afin de proposer une classification et une explication des différentes configurations morphologiques observées à l'échelle du golfe du Lion. Ce concept diverge des classifications actuelles qui se basent uniquement sur des paramètres morphologiques ou hydrodynamiques.

L'imagerie LIDAR offre en outre une vue statique instantanée de la morphologie sur un vaste ensemble géographique. Avec la réitération de cette acquisition, l'emploi de l'outil doit déboucher sur une perception dynamique. Le levé d'août 2009 permet déjà d'entrevoir la possibilité d'une telle exploitation morphodynamique. En particulier, l'observation de l'éloignement des barres à la côte conduit à limiter l'influence exclusive de facteurs statiques ou constants sur leur mode de formation, tels que la profondeur, le sens de la dérive littorale ou le caractère festonné/linéaire de la barre externe. La compréhension de l'évolution des barres sera par conséquent grandement améliorée par l'exploitation de l'imagerie LIDAR.

La précision des images LIDAR fournit également d'utiles indications en recherche appliquée côtière. En particulier les observations faites autour des ouvrages permettent de porter un jugement sur leur état, leur efficacité ou leur pertinence. Ainsi, pour la première fois, les gestionnaires bénéficieront d'un réel diagnostic d'ensemble de l'impact des ouvrages artificiels sur l'interruption ou la perturbation du transit sédimentaire en Languedoc-roussillon.

\section{Références bibliographiques}

BARUSSEAU J.P., SAINT-GUILY.B. (1981). Disposition, caractères et mode de formation des barres d'avant-côte festonnées du littoral du Languedoc-Roussillon (France). Ocea. Acta., 4, 3, pp 297-304.

CERTAIN R. (2002). Morphodynamique d'une côte sableuse microtidale à barres : le golfe du Lion (Languedoc-Roussillon). Doctorat de l'Université de Perpignan, 200 p. CERTAIN R., BARUSSEAU J.P. (2005). Conceptual modeling of sand bars morphodynamics for a microtidal beach (Sète, France). Bull. Soc. Géol. Fr., 176 (4), pp 343-354. doi:10.2113/176.4.343

SHORT A.D., AAGAARD T. (1993). Single and multi-bar beach change models. Journal of Coastal Research, Special Issue No. 15, pp 141-157.

SHRESTHA R.L., CARTER W.E., SARTORI M., LUZUM B.J., SLATTON K.C. (2005). Airborne Laser Swath Mapping: quantifying changes in sandy beaches over time scales of weeks to years. ISPRS Journal of Remonte Sensing, 59, pp 222-232. doi:10.1016/j.isprsjprs.2005.02.009

VANROYE C. (2009). Evolution des pratiques de suivi topo-bathymétrique en Languedoc-Roussillon: l'utilisation du LIDAR. Conférence Méditerranéenne Côtière et Maritime, Hammamet, Tunisie, pp 311-314. doi:10.5150/cmcm.2009.076-3 